\title{
Coherent Optical DFT-Spread OFDM
}

\author{
Fanggang Wang and Xiaodong Wang \\ Electrical Engineering Department, Columbia University, New York, NY 10027, USA \\ Correspondence should be addressed to Xiaodong Wang, wangx@ee.columbia.edu
}

Received 7 July 2010; Accepted 19 January 2011

Academic Editor: Ci-Ling Pan

Copyright () 2011 F. Wang and X. Wang. This is an open access article distributed under the Creative Commons Attribution License, which permits unrestricted use, distribution, and reproduction in any medium, provided the original work is properly cited.

\begin{abstract}
We consider application of the discrete Fourier transform-spread orthogonal frequency-division multiplexing (DFT-spread OFDM) technique to high-speed fiber optic communications. The DFT-spread OFDM is a form of single-carrier technique that possesses almost all advantages of the multicarrier OFDM technique (such as high spectral efficiency, flexible bandwidth allocation, low sampling rate, and low-complexity equalization). In particular, we consider the optical DFT-spread OFDM system with polarization division multiplexing (PDM) that employs a tone-by-tone linear minimum mean square error (MMSE) equalizer. We show that such a system offers a much lower peak-to-average power ratio (PAPR) performance as well as better bit error rate (BER) performance compared with the optical OFDM system that employs amplitude clipping.
\end{abstract}

\section{Introduction}

The high-throughput data transmission over long-haul fiber optic systems is of considerable current interest. To maximize the spectral efficiency, polarization multiplexing and coherent detection have become the key enabling technologies for high-speed fiber optic communication systems [1]. However, physical impairments such as the chromatic dispersion (CD), the polarization mode dispersion (PMD), and the polarization dependent loss (PDL) become more severe as the bandwidth and data rate increase. The orthogonal frequency-division multiplexing (OFDM) technique has been widely adopted to cope with the frequency-selective fading of multipath channels in wireless communications, and it has been recently introduced to fiber optic systems for high-speed data transmission [2]. In OFDM systems, the frequency-domain equalization is employed with a singletap equalizer at each tone, which significantly reduces the computational complexity compared with the time-domain equalization in single-carrier systems. However, one major disadvantage of the OFDM system is the high peak-toaverage power ratio (PAPR). To address this issue, the DFTspread OFDM has been developed as an alternative wireless access technique, and it has been adopted as the uplink air interface of 3rd generation partnership project long-term evolution (3GPP LTE) [3].
In [4] it is observed that the impact of nonlinearity on a link with periodical dispersion compensation is significantly larger than that on a link without inline dispersion compensation, which makes the application of OFDM to the existing infrastructure questionable. In addition, it is also noted that, in a periodic dispersion map, reducing PAPR at the transmitter might significantly improve the nonlinear tolerance of the transmission link. In order to avoid the high cost associated with mitigating the nonlinear impairments caused by the high PAPR in optical OFDM systems, in this paper, we consider the coherent optical DFT-spread OFDM to lower PAPR at the transmitter. In Section 2 we describe the optical DFT-spread OFDM system that employs polarization division multiplexing (PDM) and coherent detection. The receiver demodulation is discussed in Section 3. In Section 4 we present simulations results. Section 5 concludes the paper.

\section{The Coherent Optical DFT-Spread OFDM System with PDM}

2.1. System Descriptions. A block diagram for the optical DFT-spread OFDM system with PDM is shown in Figure 1. We transmit and receive signals on both polarizations which effectively results in a $2 \times 2$ multiple-input multiple-output 


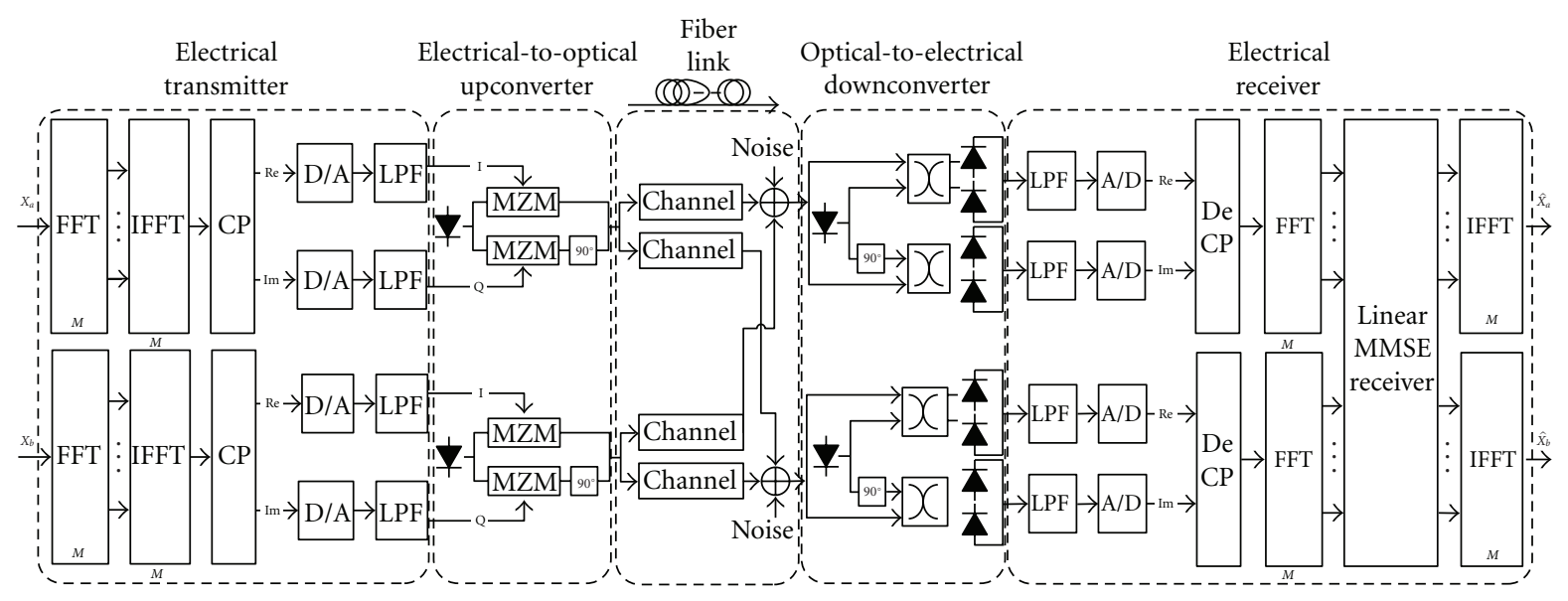

FIGURE 1: Block diagram for an optical DFT-spread OFDM system with PDM and coherent receiver.

(MIMO) system. Consider one of the two data streams at the transmitter. The bit sequence is first mapped to the quadrature amplitude modulation (QAM) symbols. In the traditional OFDM system, an inverse discrete Fourier transform (IDFT) is directly applied to the QAM symbols. In the DFT-spread OFDM system, on the other hand, a DFT is first applied to the QAM symbols and then followed by the IDFT operation. After inserting the cyclic prefix (CP) symbols, the electrical signal is passed through the digital-to-analog converter and the low-pass filter and then upconverted to the optical signal. The optical signal traverses in the longhaul fiber link comprising of multiple spans to reach the destination. At the receiver the optical signal is downconverted to the electrical signal, which is then low-pass filtered and passed through the analog-to-digital converter. After removing the $\mathrm{CP}$ and performing the DFT on the signals at each polarization, the two coupled frequency-domain received signals over $M$ subcarriers are obtained. We then perform a tone-by-tone MIMO equalization to decouple the two signal streams. Finally, the IDFT is performed on each decoupled signal stream to recover the corresponding transmitted QAM symbol stream.

Optical OFDM system has similar block diagram as shown in Figure 1 but without the DFT or IDFT modules marked as dark blocks. Intuitively, the OFDM system has a high PAPR since after IDFT at the transmitter multiple input QAM symbols could be phase-synchronously added together and therefore causes high signal amplitude (peak power). The high PAPR of the signal decreases the system power efficiency and makes the transmission more susceptible to nonlinear impairments of the fiber link. On the other hand, the DFT and IDFT cancel each other and thus the DFTspread OFDM system is essentially a single-carrier technique which in general has a much lower PAPR. However, note that the optical DFT-spread OFDM considered here is fundamentally different from the traditional single-carrier scheme [5], in that the former exhibits the advantages of both the single-carrier system (i.e., low PAPR) and the OFDM system (i.e., tone-by-tone single tap equalizer).
2.2. MIMO Channel Model for PDM. For the long-haul optical fiber transmission, the fiber link comprises $n_{E}$ fiber spans. We consider a periodic dispersion map on existing $10 \mathrm{~Gb} / \mathrm{s}$ infrastructure. In this case, the high PAPR caused by dispersion is trivial, and we therefore focus on reducing the PAPR at the transmitter. We consider three typical distortions in the fiber channel, CD, first-order PMD, and PDL. The $2 \times 2$ transfer function at subcarrier $m$ corresponding to a DFT-spread OFDM symbol is given by [5]

$$
\mathbf{H}_{m}=e^{j \phi} e^{j \Phi_{D}\left(f_{m}\right)} \mathbf{T}_{m}
$$

where $\phi$ is a common phase error (CPE) noise owing to the phase noises that varies for different OFDM symbols. In (1) $\Phi_{D}\left(f_{m}\right)$ is the phase dispersion owing to $\mathrm{CD}$ and is given by

$$
\Phi_{D}\left(f_{m}\right)=\frac{\pi c D L f_{m}^{2}}{f_{c}^{2}}, \quad \text { with } f_{m}=\frac{m-1}{t_{s}}
$$

where $t_{s}$ is the actual time duration of one DFT-spread OFDM symbol not including the cyclic prefix, $c$ is the speed of the light, $D$ is the CD parameter in unit of $\mathrm{ps} / \mathrm{pm} / \mathrm{km}$; $L$ is the total length of the multispan fiber link, and $f_{c}$ is the carrier frequency of the laser. Here we assume that the periodic dispersion map is employed, and the CD is completely compensated for at each span. Hence $\mathbf{T}_{m}$ in (1) is the Jones matrix with the dispersion compensation fiber (DCF) inserted at each stage, given by

$$
\begin{aligned}
\mathbf{T}_{m}= & \prod_{l=1}^{n_{E}} e^{-j\left(\Phi_{D}\left(f_{m}\right) / n_{E}\right)}\left[\begin{array}{ll}
1 & 0 \\
0 & k_{l}
\end{array}\right] \cdot\left[\begin{array}{cc}
e^{j \pi\left(f_{c}+f_{m}\right) \tau_{l}} & 0 \\
0 & e^{-j \pi\left(f_{c}+f_{m}\right) \tau_{l}}
\end{array}\right] \\
& \cdot\left[\begin{array}{cc}
\cos \theta_{l} & \sin \theta_{l} \\
-\sin \theta_{l} & \cos \theta_{l}
\end{array}\right],
\end{aligned}
$$

where $k_{l}$ denotes the attenuation factor of PDL, $\tau_{l}$ is the differential group delay (DGD), and $\theta_{l}$ is the uniformly random rotation angle [6]. For each span, the DGD is 
a random variable following the Maxwellian distribution. However, in the same span, the DGDs of different subcarriers are the same.

\section{The Linear MMSE Coherent Receiver}

Let $\mathbf{F}$ be the $M \times M$ DFT matrix with its element given by

$$
\mathbf{F}_{\alpha, \beta}=\left(\frac{1}{\sqrt{M}}\right) e^{-j 2 \pi(\alpha-1)(\beta-1) / M} .
$$

Denote the transmitted QAM symbols on all subcarriers along the $k$ th polarization as $\mathbf{s}^{(k)} \triangleq\left[s_{1}^{(k)}, s_{2}^{(k)}, \ldots, s_{M}^{(k)}\right]^{T}$. As shown in Figure 1, a DFT operation is first applied to $\mathbf{s}^{(k)}$, to obtain

$$
\mathbf{x}^{(k)} \triangleq\left[x_{1}^{(k)}, x_{2}^{(k)}, \ldots, x_{M}^{(k)}\right]^{T}=\mathbf{F} \mathbf{s}^{(k)},
$$

which is effectively the input to a traditional OFDM system. At the receiver, we assume that the frequency and time offset can be perfectly estimated and compensated before detection. Then the received signal on the $m$ th subcarrier is given by

$$
\mathbf{y}_{m}=\mathbf{H}_{m} \mathbf{x}_{m}+\mathbf{v}_{m}, \quad m=1, \ldots, M,
$$

where $\mathbf{x}_{m} \triangleq\left[x_{m}^{(1)}, x_{m}^{(2)}\right]^{T}$ and $\mathbf{v}_{m} \sim \mathcal{N}_{c}\left(\mathbf{0}, \sigma^{2} \mathbf{I}\right)$.

To demodulate the symbol vector $\mathbf{s}^{(k)}$, we first estimate the DFT-spread symbol $\mathbf{x}^{(k)}$ and then recover the data symbol vector $\mathbf{s}^{(k)}$ by an IDFT. In particular, a linear MMSE estimate of $\mathbf{x}_{m}$ based on $\mathbf{y}_{m}$ in (6) is given by [7]

$$
\begin{aligned}
\hat{\mathbf{x}}_{m} & =\boldsymbol{\Gamma} \mathbf{H}_{m}^{\dagger}\left(\sigma^{2} \mathbf{I}+\mathbf{H}_{m} \mathbf{H}_{m}^{\dagger}\right)^{-1} \mathbf{y}_{m} \\
& =\boldsymbol{\Gamma}\left(\sigma^{2} \mathbf{I}+\mathbf{H}_{m}^{\dagger} \mathbf{H}_{m}\right)^{-1} \mathbf{H}_{m}^{\dagger} \mathbf{y}_{m}, \quad m=1, \ldots, M,
\end{aligned}
$$

where $(\cdot)^{\dagger}$ denotes conjugate transpose and $\boldsymbol{\Gamma}$ is a diagonal matrix with $\gamma_{k, k}=1 /\left(1-\left[\left(\mathbf{I}+\mathbf{H}_{m} \mathbf{H}_{m}^{\dagger} / \sigma^{2}\right)^{-1}\right]_{k, k}\right)$. Note that the tone-by-tone linear MMSE equalization in (7) involves inverting $2 \times 2$ matrices, and hence the computational complexity is not significant. Denote $\hat{\mathbf{x}}^{(k)} \triangleq\left[\hat{x}_{1}^{(k)}, \ldots, \hat{x}_{M}^{(k)}\right]^{T}$. We finally apply the IDFT on $\widehat{\mathbf{x}}^{(k)}$ to obtain the estimated data symbols

$$
\widehat{\mathbf{s}}^{(k)}=\mathbf{F}^{\dagger} \hat{\mathbf{x}}^{(k)}, \quad k=1,2 .
$$

\section{Simulation Results}

In this section we provide simulation results to compare the optical DFT-spread OFDM system with the optical OFDM system in terms of both the PAPR performance and the bit error rate (BER) performance. The number of subcarriers is $M=256$. A long-haul fiber optic system is considered with $n_{E}=12$ cascaded spans (each of length $L=80 \mathrm{~km}$ ). The laser wavelength is $\lambda=1.55 \mu \mathrm{m}$. The $\mathrm{CD}$ parameter is $D=$ $17 \mathrm{ps} / \mathrm{nm} / \mathrm{km}$. We assume that periodic dispersion map is employed, and the CD is compensated for by DCF after each

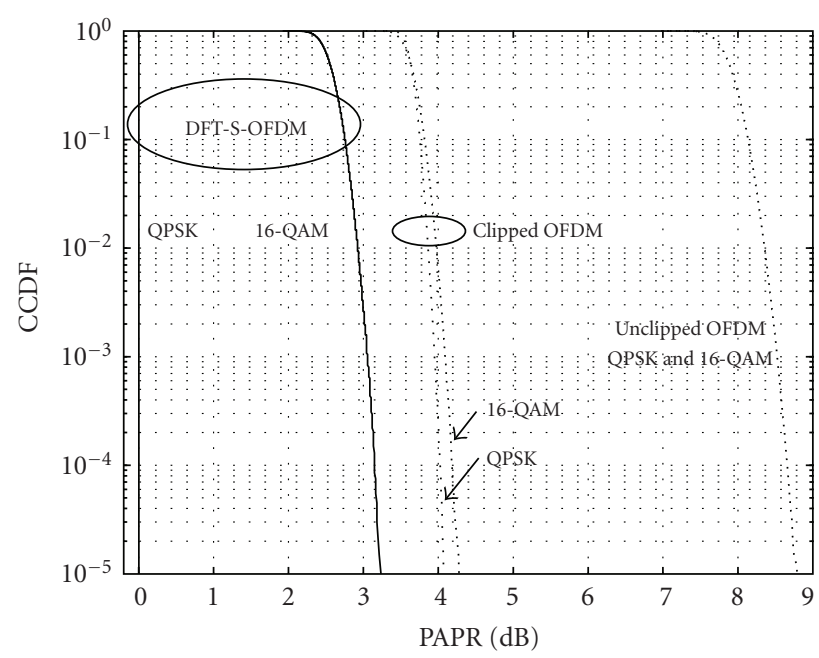

FIGURE 2: Comparison of the complementary cumulative distribution function (CCDF) of the PAPR.

span. The DGD parameter is $D_{p}=0.15 \mathrm{ps} / \sqrt{\mathrm{km}}$. The mean value of DGD is $\sqrt{8 /(3 \pi)} D_{p} \sqrt{n_{E} L}$. A typical PDL is $0.1 \mathrm{~dB}$, where PDL $[\mathrm{dB}]=-20 \log \left(k_{l}\right)$. We consider both QPSK and 16-QAM modulations. The data rate is $25 \mathrm{G}$ symbols/s, that is, $100 \mathrm{~Gb} / \mathrm{s}$ for QPSK and $200 \mathrm{~Gb} / \mathrm{s}$ for 16QAM.

Figure 2 illustrates the PAPR performance of the two systems for QPSK and 16-QAM. It is seen that the OFDM system exhibits a much higher PAPR than the DFT-spread OFDM system. In general 16-QAM has a higher PAPR than QPSK. However, their PAPRs are similar in the OFDM system. Amplitude clipping is a typical method to lower the PAPR in OFDM systems. We used a clipping ratio (CR) of $3 \mathrm{~dB}$, defined as $\mathrm{CR}=20 \log _{10}(A / P)$, where $P$ is the power of transmitted signal and $A$ is the maximum transmitted signal magnitude. It is seen that clipping can indeed significantly reduce the PAPR in the OFDM system; however, the PAPR in the clipped OFDM system is still much larger than that of the DFT-spread OFDM system. On the other hand, clipping substantially degrades the bit error rate (BER) performance. As shown in Figure 3, the DFT-spread OFDM and the OFDM systems have the same BER performance. But after the $3 \mathrm{~dB}$ clipping on the OFDM signal, a $0.8 \mathrm{~dB}$ loss is incurred at the BER of $10^{-3}$ for QPSK, and for 16QAM the loss due to clipping is about $8 \mathrm{~dB}$. In summary, compared with the traditional optical OFDM system that employs amplitude clipping, the DFT-spread OFDM system offers both a lower PAPR and a better BER performance.

Discussions. Note that the effect of nonlinearity depends on the instantaneous power of the signal according to Schrodinger's equation [8]. It is shown in Figure 2 that the PAPR performance of the DFT-spread OFDM signal is better than both the clipped and unclipped OFDM signals. This means that, for a given average transmit power, the peak power of both the clipped and unclipped OFDM signals will be larger than that of the DFT-spread OFDM 


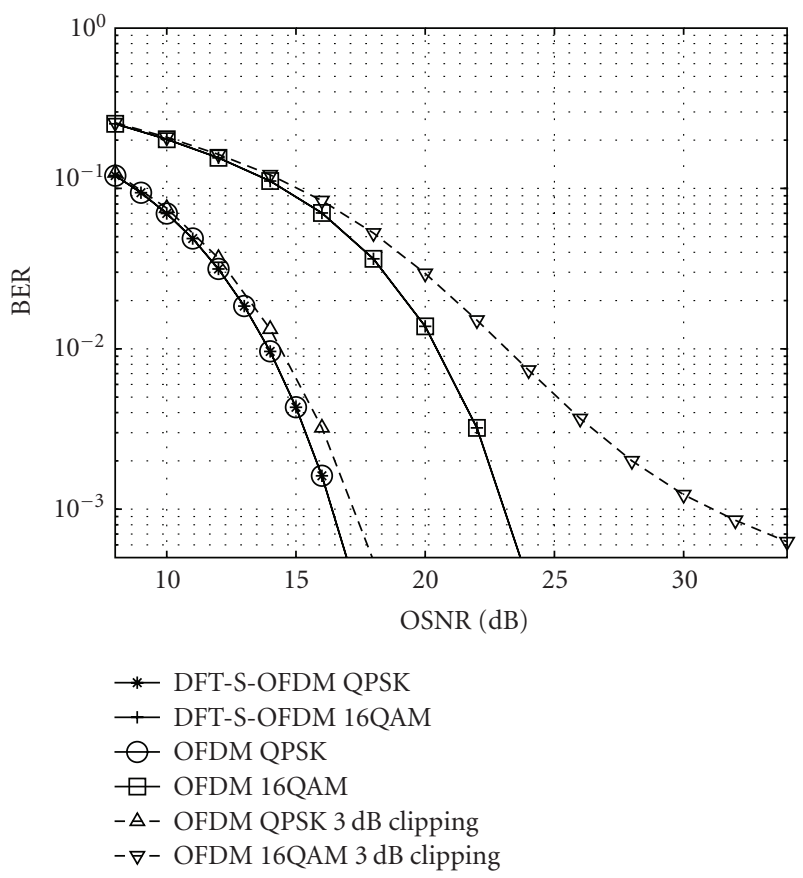

FIgURE 3: BER comparison.

signals, and hence they are more susceptible to nonlinear distortion than the DFT-spread OFDM signals. On the other hand, various effective nonlinear impairment compensation methods have been proposed [9]. Hence, one can envision that, if these techniques are applied to the system, only linear channel distortion needs to be considered, under which we have shown that the DFT-spread OFDM offers better BER performance than the clipped OFDM.

\section{Conclusions}

We have considered the optical DFT-spread OFDM system with polarization division multiplexing and coherent detection. Compared with the conventional single-carrier systems, the DFT-spread OFDM system has the advantages of flexible bandwidth allocation, high spectral efficiency, and low sampling rate, and low-complexity equalization. Compared with the optical OFDM system with amplitude clipping, the DFT-spread OFDM system offers both better BER performance and a much lower PAPR, with little attendant increase in the transceiver complexity.

\section{References}

[1] E. Ip, A. P. T. Lau, D. J. F. Barros, and J. M. Kahn, "Coherent detection in optical fiber systems," Optics Express, vol. 16, no. 2, pp. 753-791, 2008.

[2] J. Armstrong, "OFDM for optical communications," Journal of Lightwave Technology, vol. 27, no. 3, pp. 189-204, 2009.

[3] ETSI Std., "Evolved universal terrestrial radio access (EUTRA) and evolved universal terrestrial radio access network (E-UTRAN); overall description; stage 2," 3GPP TS36.300, Rev. 9.1.0, 2009.
[4] K. Forozesh, S. L. Jansen, S. Randel, I. Morita, and H. Tanaka, "The influence of the dispersion map in coherent optical OFDM transmission systems," in IEEE/LEOS Summer Topical Meetings, pp. 135-136, July 2008.

[5] W. Shieh, X. Yi, Y. Ma, and Q. Yang, "Coherent optical OFDM: has its time come?” Journal of Optical Networking, vol. 7, no. 3, pp. 234-255, 2008.

[6] F. N. Hauske, M. Kuschnerov, B. Spinnler, and B. Lankl, "Optical performance monitoring in digital coherent receivers," Journal of Lightwave Technology, vol. 27, no. 16, pp. 3623-3631, 2009.

[7] N. Prasad, S. Wang, and X. Wang, "Efficient receiver algorithms for DFT-spread OFDM systems," IEEE Transactions on Wireless Communications, vol. 8, no. 6, pp. 3216-3225, 2009.

[8] G. P. Agrawal, Nonlinear Fiber Optics, Academic Press, San Diego, Calif, USA, 3rd edition, 2001.

[9] E. Mateo, L. Zhu, and G. Li, "Impact of XPM and FWM on the digital implementation of impairment compensation for WDM transmission using backward propagation," Optics Express, vol. 16, no. 20, pp. 16124-16137, 2008. 

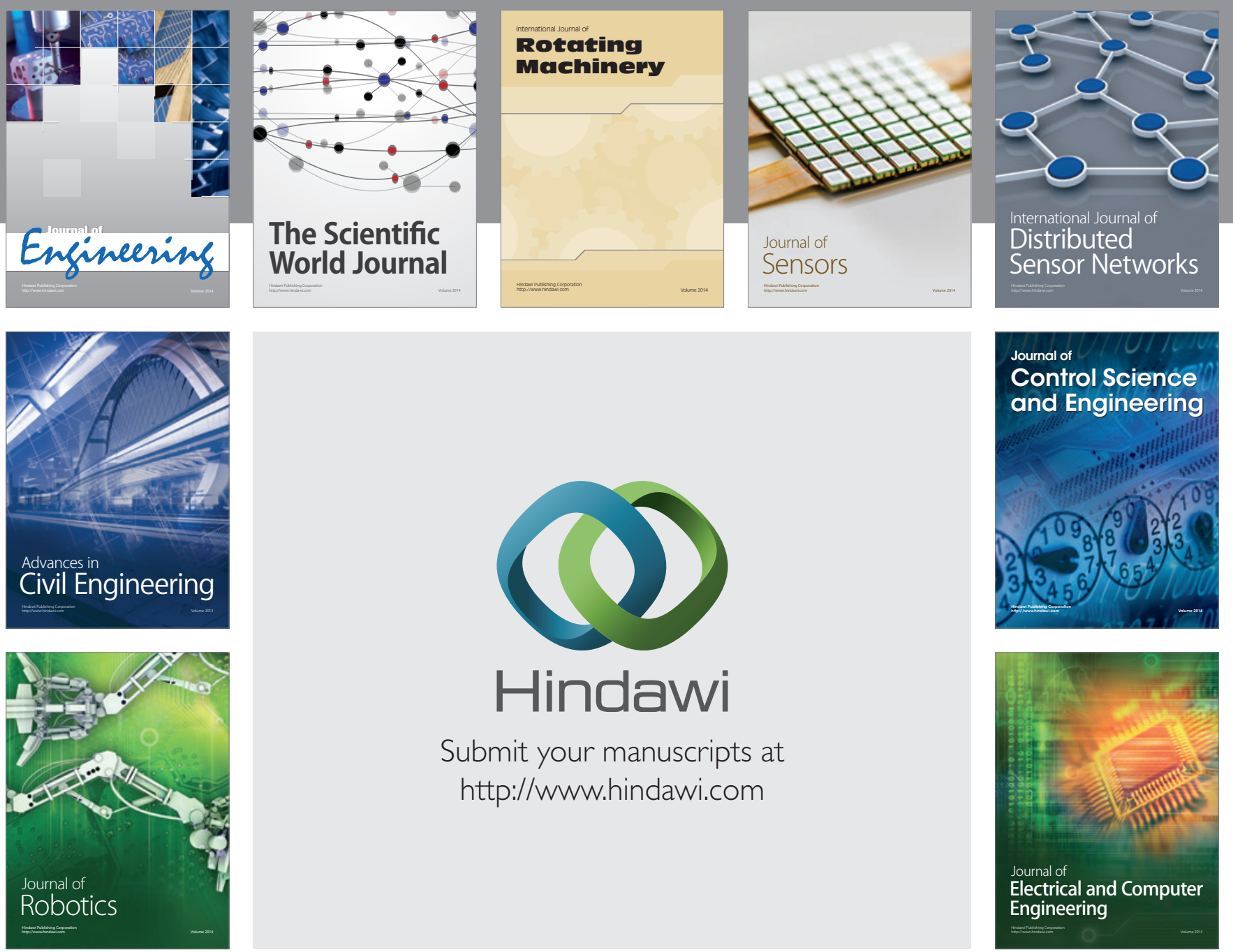

Submit your manuscripts at

http://www.hindawi.com
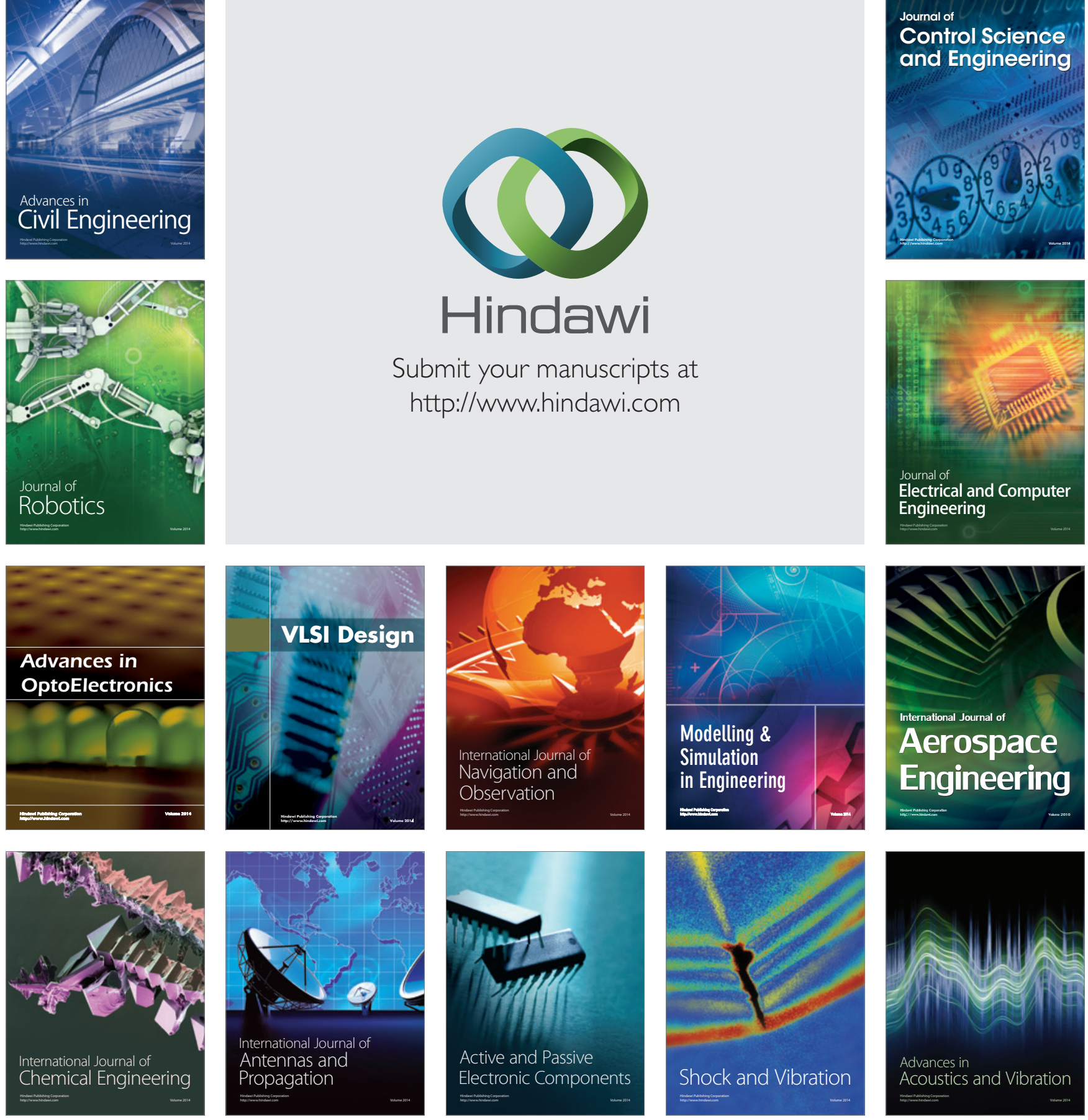